\title{
Velocity oscillations and stop-go cycles: The trajectory of an object settling in a cornstarch suspension
}

\author{
Stefan von Kann, Jacco H. Snoeijer, and Devaraj van der Meer \\ Physics of Fluids Group, University of Twente, P. O. Box 217, 7500 AE Enschede, The Netherlands
}

(Received 30 November 2012; published 1 April 2013)

\begin{abstract}
We present results for objects settling in a cornstarch suspension. Two surprising phenomena can be found in concentrated suspensions. First, the settling object does not attain a terminal velocity but exhibits oscillations around a terminal velocity when traveling through the bulk of the liquid. Second, close to the bottom, the object comes to a full stop but then reaccelerates before coming to another stop. This cycle can be repeated up to 6 or 7 times before the object reaches the bottom to come to a final stop. For the bulk, we show that shear-thickening models are insufficient to account for the observed oscillations and that the history of the suspension needs to be taken into account. A hysteretic model, that goes beyond the traditional viscoelastic ones, describes the experiments quite well but still misses some details. The behavior at the bottom can be modeled with a minimal jamming model.
\end{abstract}

DOI: 10.1103/PhysRevE.87.042301

PACS number(s): 82.70.Kj, 45.70.Vn, 47.50.-d, 47.57.ef

\section{INTRODUCTION}

A suspension is a heterogeneous fluid that contains dispersed solid particles which are large enough to sediment over time when undisturbed. They are literally found all around us and the flow of dense suspensions is important in nature (mud slides), industry (paint), and even health care (blood flow) [1]. In spite of their significance, the flow of these dense suspensions remains far from understood. In previous studies, people have used methods inspired by classical rheology and typically characterized these materials in terms of a constitutive relation of stress versus shear rate [2-7]. A general result is that, when increasing the shear rate, dense suspensions first tend to become less viscous (shear thinning) and subsequently shear thicken.

At very high strain rates the particles inside a dense suspension may jam, i.e., particles may form force networks that temporarily inhibit (part of) the suspension to flow. The concept of jamming - which will play a major role in the interpretation of the experiments discussed in this paper-is well known from the statics and dynamics of dry granular materials $[8,9]$, where it was shown to be connected to the concepts of random loose and close packing [10]. When particles go away from a spherical shape, their random close packing fractions go down from approximately 0.64 for hard spheres [11] to around 0.45 for various starches [12] and even lower for more irregularly shaped particles [13].

Probably the most conspicuous example of a dense suspension is formed by a high concentration of cornstarch in water, also known as oobleck or ooze. In earlier work, rheology experiments with cornstarch suspensions have revealed the existence of a mesoscopic length scale [6,14], a shear-thinning regime that terminates in a sudden shear thickening [15], a dynamic jamming point [4], and fracturing [16]. In an experiment that goes beyond the classical rheological ones, Merkt et al. [17] observed that stable oscillating holes can be formed in a thin layer of cornstarch suspension when shaken vertically at certain frequencies and amplitudes [17]. These holes were subsequently described using a phenomenological model based on a hysteretic constitutive equation [18]. In other dense suspensions, Ebata et al. found growing and splitting holes $[19,20]$, where the first are contributed to a convectionlike flow and the latter are still not understood. Another set of remarkable observations were made for settling objects. These displayed nonmonotonic settling [21] and jamming between the object and container bottom was found $[21,22]$. At present we are, thus, still far from a detailed understanding of dense suspensions and why different suspensions behave differently.

The current paper will provide a full exposition and expansion of the work presented in Ref. [21]: We subject a cornstarch suspension to a basic experiment, in which we observe and describe the settling of objects in a deep bath of suspension. The settling dynamics exhibits two remarkable features that are not observed in other types of liquids but also not in other dense suspensions. In the bulk, we find that the object velocity is oscillating in addition to going towards a terminal value. Near the bottom we observe a second phenomenon: The object comes to a full stop before the bottom but then accelerates again, and this stop-go cycle can repeat up to 7 times. Although nonmonotonic settling has been observed in various other systems, like stratified [23] and (visco-)elastic [24,25] liquids, we will show that both bulk and bottom behavior in cornstarch fundamentally differ. We study a wide range of experimental parameters and suspensions to get detailed insight in these phenomena, discuss several candidates for the (phenomenological) modeling of the observed phenomena, and evaluate their appropriateness.

This paper is organized as follows. In Sec. II we discuss the experimental setup and some data analysis tools. Subsequently, the main experimental observations are presented in Sec. III, where the influence of various parameters such as the concentration of the cornstarch suspension, the object mass, the object shape, and the container size are discussed. Section IV focuses on the bulk oscillations by presenting its particular experimental characteristics and by subsequently discussing the validity of several modeling approaches. The stop-go cycles at the bottom obtain a similar treatment in Sec. V, a large part of which is devoted to the comparison of a jamming model and the experiments, expanding the material presented in Ref. [21]. Finally, in Sec. VI, we briefly discuss the 
settling dynamics in other particulate suspensions and Sec. VII concludes the paper.

\section{EXPERIMENTAL SETUP}

The experimental setup is shown in Fig. 1(a). Objects were dropped into either a vertical perspex container of size $12 \times 12 \times 30 \mathrm{~cm}^{3}$ or a cylindrical glass container with a diameter of $5.0 \mathrm{~cm}$, containing a dense mixture of particles and liquid. For the latter, we use either demineralized water or an aqueous solution of cesium chloride $(\mathrm{CsCl})$ with a density of $\sim 1600 \mathrm{~kg} / \mathrm{m}^{3}$, which matches the density of the cornstarch particles. Experimental results actually showed negligible differences between the density matched and the unmatched liquid, provided that the latter has to be stirred well prior to the experiment to counteract sedimentation. The cornstarch particles [Fig. 1(b)] are irregularly shaped and have an approximately flat size distribution in the range of 5-20 $\mu \mathrm{m}$, i.e., small and large particles are present in approximately equal numbers.

The settling objects that were used in this study are stainless steel balls $\left(\rho=8000 \mathrm{~kg} / \mathrm{m}^{3}\right)$, with diameters of 1.6 and $4.0 \mathrm{~cm}$, a $4.0-\mathrm{cm}$ ping-pong ball, and a $1.5-\mathrm{cm}$ diameter hollow cylinder with a flat bottom and a length longer than the liquid bath depth. The latter two can be filled with bronze beads to vary their mass: For the ping-pong ball, the buoyancy-corrected mass $\left(\mu=m_{\text {sphere }}-\rho_{S} V\right.$ of the objects, where $\rho_{S}$ is the suspension density and $V$ is the submersed volume) could be varied from 0 to $137 \mathrm{~g}$ and the actual mass $m$ of the cylinder was varied from 40 (empty cylinder) to
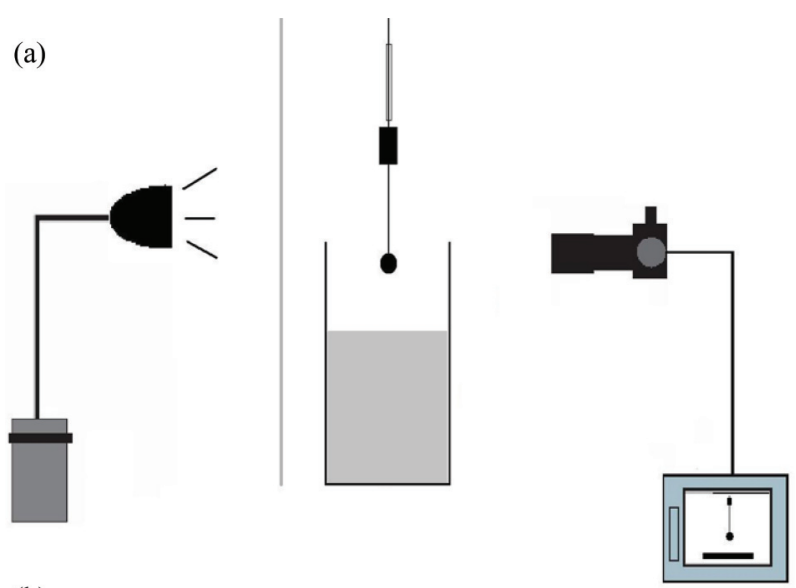

(b)

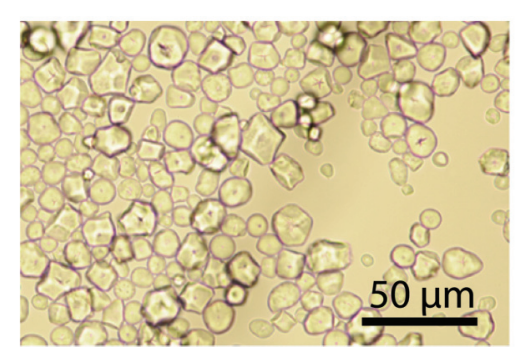

FIG. 1. (Color online) (a) Schematic view of the setup with, from left to right, a light source and diffusing plate, the container filled with suspension, above that the object with tracers attached, and a high-speed camera. (b) Microscopic picture of the cornstarch, used in the experiments.

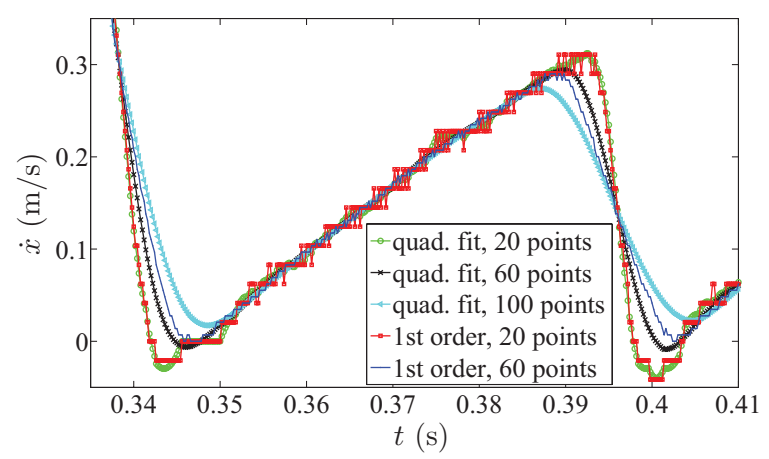

FIG. 2. (Color online) The velocity $\dot{x}$ of the settling object versus time $t$, determined by two different methods, namely (i) using a local quadratic fit and (ii) employing a first-order difference. The two methods are shown for different time intervals of the fitting procedures, namely $4.0,12$, and $20 \mathrm{~ms}$, to illustrate the trade-off when choosing between higher spatial or temporal resolution.

$120 \mathrm{~g}$. For the cylinder, the buoyancy-corrected mass is not constant over time: The buoyancy increases when the cylinder penetrates deeper into the cornstarch, such that $\mu$ decreases over time. The results for the cylinder will therefore be given in terms of the actual mass.

To measure the trajectory of the objects inside the opaque suspension, we follow tracers on a thin, rigid metal wire that is attached to the top of the object (as in Ref. [26]) with a high-speed camera imaging at 5000 frames per second. The mass of the wire and the resulting buoyancy of the immersed wire are negligible compared to the larger object to which it is attached. Namely, the mass of the wire is less than $1 \mathrm{~g}$ and the immersed tail volume is smaller than 0.1 times the volume of the smallest object that was used. The velocity and acceleration are determined from the trajectories at each time $t$, using either (i) a local quadratic fit around $t$ or (ii) a direct first- and second-order difference, both determined over a time interval of $12 \mathrm{~ms}$ (corresponding to 60 measurement points). The difference between both methods and the influence of the interval are illustrated in Fig. 2, where we show the results of both procedures for the velocity of the object during a particularly sensitive part of the trajectory with abrupt jumps in the velocity. Clearly, when an interval of $4.0 \mathrm{~ms}$ (corresponding to 20 measurement points) is used, the signal suffers from pixel noise due to the limited spatial resolution of our camera. For an interval of $20 \mathrm{~ms}$ (100 points) we observe that a lot of information is lost: The abrupt decreases in velocity flatten out, and the maximum and minimum velocities are resolved insufficiently. For the above reasons, the time interval was fixed to $12 \mathrm{~ms}$, as it showed the best trade-off when choosing between losing pixel noise due to limits in spatial resolution and losing temporal resolution. In addition, the local quadratic fit leads to a more accurate determination of the acceleration than the method using the second-order difference.

\section{EXPERIMENTAL OBSERVATIONS}

In this section we will present the main experimental observations. We will start by comparing the settling in a viscous Newtonian liquid to the settling in a cornstarch suspension and show that the behavior of the latter is highly 


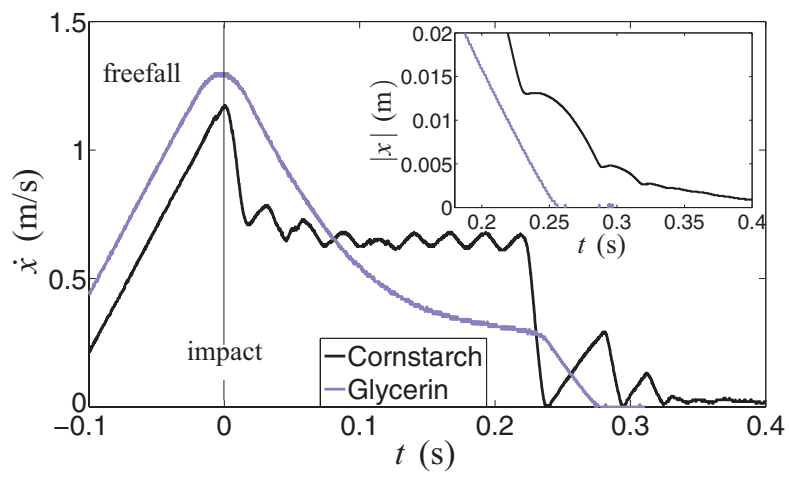

FIG. 3. (Color online) The settling velocities $\dot{x}$ of a steel sphere with a diameter of $0.5 \mathrm{~cm}$ in glycerine [blue (gray) line] and a steel sphere of diameter $1.6 \mathrm{~cm}$ in a cornstarch suspension with $\phi=0.41$ (black line) as a function of time $t$. The inset shows the last part of the actual trajectory, clearly showing the stop-go cycles near the bottom in cornstarch in the position versus time curve.

non-Newtonian. This will be followed by a discussion of the effects that various parameters have on the experiment.

\section{A. General observations}

In Fig. 3 we plot the time evolution of the velocity of a steel sphere for two different impact experiments: one on glycerin and the other on a cornstarch suspension. The sphere is released and accelerates up to $t=0$, which is the moment of impact. For glycerin [blue (gray) line], a Newtonian liquid, we find the expected behavior for such a liquid: The sphere gradually decelerates and exponentially decays towards a terminal velocity, which is determined by the object and the liquid properties. The experiment ends when the object stops at the container bottom. When looking at the dense cornstarch suspension (black line), we observe some remarkable phenomena: On impact, we first see an abrupt decrease towards a lower velocity, which in recent experiments by Waitukaitis et al. [27] was explained to be caused by jamming of the suspension on impact. Subsequently, instead of monotonously approaching a terminal velocity, there appear velocity oscillations around this terminal value: The object alternately goes through periods of acceleration and deceleration. The oscillations show no sign of damping out in the time span that is available to us experimentally. These extraordinary oscillations are quite unlike oscillations that have, e.g., been observed in viscoelastic fluids, for which the amplitude rapidly decays. We refer to the oscillations in our experiment as bulk oscillations, to distinguish them from the second phenomenon: Instead of stopping at the bottom, the object actually comes to a sudden, full stop at about $10 \mathrm{~mm}$ above the bottom. Surprisingly, instead of just staying there, the object subsequently reaccelerates, only to come to another stop a little closer to the bottom. This process repeats itself several times until the bottom is reached. From here onward, we will call these phenomena stop-go-cycles.

As the density matching of such a large bath requires a forbiddingly large amount of salt, we repeated the experiment in an unmatched suspension. Although the cornstarch particles are heavier than the liquid, the settling of particles is negligible for at least several minutes, as we were able to ascertain by performing experiments after different waiting times after stirring, which showed identical behavior. Most of the experiments presented in the current work are, therefore, performed after stirring well but without density matching.

Before taking a more detailed look at the origin of both effects, we will first discuss how these bulk oscillations and stop-go cycles are influenced by changing the experimental parameters of the liquid bath and the settling object. We find only minor changes when varying the impact velocity and the bath depth. The onset of the bulk oscillations is depending on the impact velocity and may sometimes not occur in shallower layers, as the necessary mean velocity is not reached. The stop-go cycles are independent on these parameters; only for shallower layers will the first stop be closer to the surface of the suspension. The response of a thin layer of cornstarch suspension in between an object and a substrate is covered in Liu et al. [22] and is consistent with our findings.

\section{B. Packing fraction}

To determine the influence of the packing fraction, we focus on results of a 1.6- $\mathrm{cm}$ stainless steel ball settling in suspensions of different packing fractions $(\phi)$, where $\phi$ is the volume occupied by the particles over the total volume of the suspension. The velocity of the ball for different concentrations is plotted in Fig. 4 as a function of time. In the plots, $t=0$ coincides with the moment of impact on the suspension.

First, we observe that the velocity of the sphere within the suspension has none of the particular characteristics for cornstarch concentrations up to volume fractions of $\phi=0.38$. The behavior is similar to what is observed for a Newtonian liquid and the only difference is the way the fluid responds on impact, where we observe a sudden decrease of the velocity. This may well be connected to compaction on impact as discussed in Ref. [27]. While increasing the concentration of cornstarch we see the velocity drop become more pronounced, which is an indication of a larger jammed region created on impact, consistent with the observations in Ref. [27]. Another observation is that the terminal velocity is smaller and appears to be reached at an earlier point in time for higher $\phi$, which can be explained from an overall increase of the apparent (or average) viscosity of the suspension.

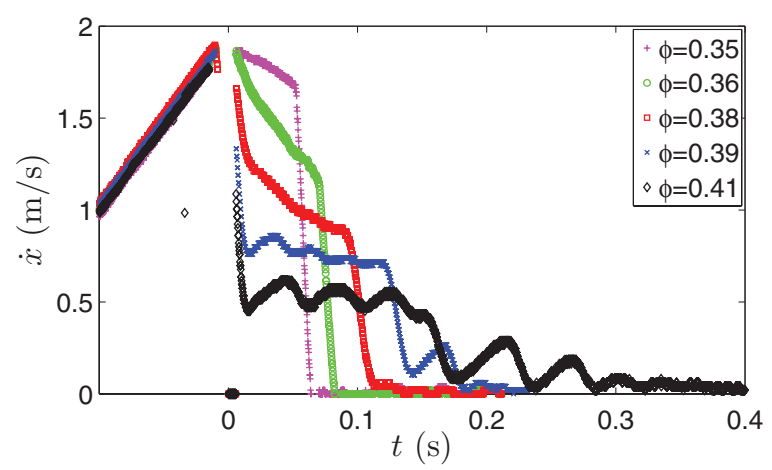

FIG. 4. (Color online) Settling velocity $\dot{x}$ of a stainless steel sphere (diameter $1.6 \mathrm{~cm}$ ) in a cornstarch suspension as a function of time $t$ and for different cornstarch packing fractions $\phi$ varying from 0.35 to 0.41 . 
When reaching $\phi=0.39$, we start to observe the nonmonotonic settling behavior that was discussed in the previous subsection: After impact, we first observe velocity oscillations in the bulk and, afterward, when the sphere approaches the bottom, the stop-go cycles. For increasing cornstarch concentration, we observe a significant increase of the amplitude of the bulk oscillations, on the one hand, and of the amplitude, the duration, and the number of stop-go cycles on the other. The frequency of the bulk oscillations seems to be less affected by $\phi$.

Clearly, both phenomena are most pronounced for high $\phi$, which is why for the remainder of this study we will will fix our bath concentration at the particularly high value $\phi=0.44$, unless specified differently.

\section{Container size}

We performed identical impact experiments with the 1.6-cm steel sphere in two different containers (one with a circular cross section of $5 \mathrm{~cm}$ and the other with a square cross section of $\left.12 \times 12 \mathrm{~cm}^{2}\right)$ containing a single batch of suspension $(\phi=$ 0.42 ) and compared the results to see whether the proximity of the side walls influences, or maybe even causes, the observed phenomena. The results are shown in Fig. 5.

Already immediately after impact the behavior deviates for the different containers: The ball decelerates in both cases but for the smaller container even comes to an almost full stop. This is likely to be caused by jamming of the suspension in a cone-shaped region below the sphere, as observed in Ref. [27]. Whereas this jammed region may move along with the sphere in the larger container, this region may extend all the way up to the wall of the smaller container, such that the sphere is not able to move down in that case.

After this initial velocity drop, both experiments reach a terminal velocity that is a bit lower for the smaller container. This can be attributed to the proximity of the container wall as well, which will increase the drag in a similar way as it would in a viscous Newtonian liquid. The bulk oscillations are discernible in both containers but are much less pronounced in the smaller one. This leads to the important conclusion that the

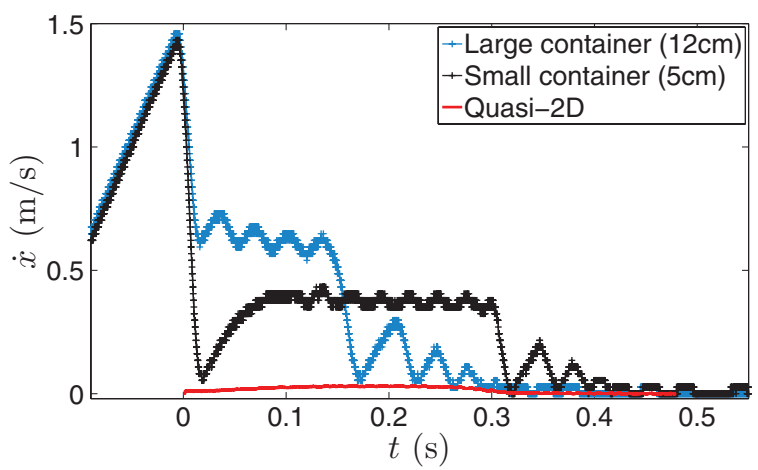

FIG. 5. (Color online) The settling velocity $\dot{x}$ versus time $t$ in a $12 \times 12 \mathrm{~cm}^{2}$ square container and a cylindrical container with a diameter of $5.0 \mathrm{~cm}$ for a ball of $1.6 \mathrm{~cm}$ diameter impacting with a velocity of $1.5 \mathrm{~m} / \mathrm{s}$ on a cornstarch suspension with a concentration of $\phi=0.42$. For comparison, the result of a cylindrical disk settling in a quasi-2D setup is added. bulk oscillations are truly a bulk effect, i.e., they are weakened by the proximity of the side walls rather than being reinforced.

By contrast, the stop-go cycles at the bottom are qualitatively the same, and only the maximum velocities that are reached during the reacceleration phase differ slightly. The smaller container again reaches somewhat lower velocities. This may, however, well be connected to the fact that the terminal velocity is smaller for the small container.

In addition to varying the container size, we repeated the experiment in a quasi-two-dimensional setup, in a rectangular container with a cross section of $100 \times 5 \mathrm{~mm}^{2}$ and a depth of $50 \mathrm{~mm}$, using a cylindrical disk with a diameter of $1.5 \mathrm{~cm}$ and a thickness of $4 \mathrm{~mm}$ as a settling object. In this experiment, we hoped to be able to discern variations in suspension concentration below the settling object. What we observed, however, was that all effects actually fully disappeared due to the large friction between the object and the lateral container walls. We added this quasi-2D experiment to Fig. 5, where it can be appreciated that the (terminal) settling velocity is only a few centimeters per second.

\section{Object mass}

Whereas in the previous subsections we discussed the influence of the bath properties on the observed phenomena, we now turn to the settling object itself. First, we consider the effect of the buoyancy-corrected mass $\left(\mu=m_{\text {sphere }}-\right.$ $4 / 3 \pi r^{3} \rho_{S}$ with $\rho_{S}$ the density of the suspension) by using a hollow ping-pong ball, with a radius $r=2.0 \mathrm{~cm}$, that can be filled with bronze beads to a mass $m_{\text {sphere }}$. This allows us to vary the difference in density between the impactor and the suspension while keeping all other parameters constant. By completely filling the ball we can reach a maximum density of $5.4 \times 10^{3} \mathrm{~kg} / \mathrm{m}^{3}$, which is around 3.5 times the suspension density but lower than the density of the steel sphere used before $\left(\rho_{\text {steel }} \approx 8.0 \times 10^{3} \mathrm{~kg} / \mathrm{m}^{3}\right)$. The resulting velocity versus time curves for these measurements can be found in Fig. 6.

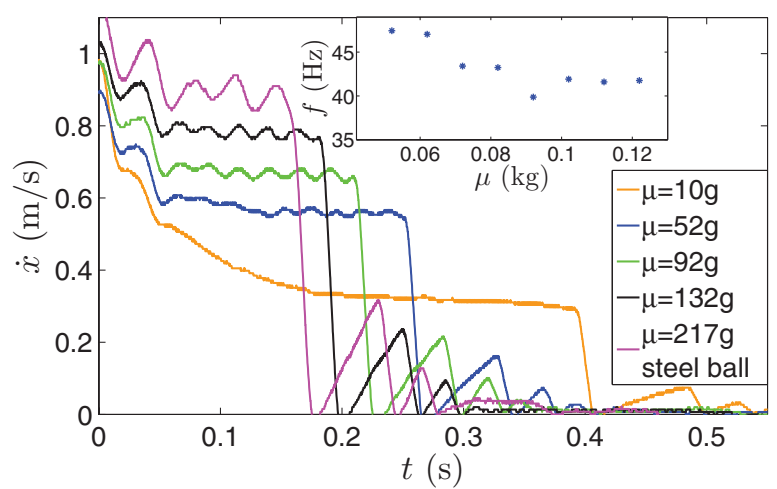

FIG. 6. (Color online) Time evolution of the velocity $\dot{x}$ of a hollow ping-pong ball filled with different masses settling in a cornstarch suspension with $\phi=0.44$. The buoyancy-corrected mass varies from $\mu=10$ to $\mu=132 \mathrm{~g}$. Also added is an experiment with a steel sphere of $\mu=217 \mathrm{~g}$, with the same diameter $(4.0 \mathrm{~cm})$ as the ping-pong ball. The inset shows the frequency of the bulk oscillations for the ping-pong ball. 
We observe no pronounced bulk oscillations (and even something that looks like an exponential decay) for the experiments with lighter spheres (up to $90 \mathrm{~g}$, corresponding to $\mu=47 \mathrm{~g}$ ). When we keep increasing the object's mass, the bulk oscillations appear. These start out at very small amplitude but increase with increasing mass. Another remarkable observation is that the oscillation period varies only slightly over the entire range of masses where the bulk oscillations are visible: While the buoyancy-corrected mass grows over a factor 2 , the oscillation frequency only shows a slight decrease of around 20\% (Fig. 6, inset).

In contrast to the bulk oscillations, the stop-go cycles are observed for all masses, even for the smallest buoyancycorrected mass of $\mu=10 \mathrm{~g}$ which corresponds to a density difference between object and suspension of only $23 \%$. The magnitude of the stop-go cycles, i.e., both the maximum velocity and the number of cycles, is found to increase with the mass of the object.

For comparison, we also used a massive steel sphere, with $r=2.0 \mathrm{~cm}$ and $\mu=217 \mathrm{~g}$. Thus, we obtain an even higher density contrast but at the expense of changing the surface of the object. During settling of this sphere, we observe the same phenomena as for the ping-pong ball. The increasing trend we found for the amplitude of the bulk oscillations and the maximum velocity and number of stop-go cycles is continued. The main difference is the fact that we measure a frequency which is a factor 1.5 lower for the bulk oscillations. This may be connected to the different structure of the surface of the object.

\section{E. Object shape}

Besides changing the mass of the object, we also varied its shape. We used a hollow cylinder with a diameter of 1.5 $\mathrm{cm}$ and a height that exceeds the depth of the cornstarch bath. This changes two aspects: First, the object has a larger contact area with the liquid, and, second, we have a flat bottom rather than a curved one. The fact that the cylinder is longer than the depth of the bath allows us to keep it aligned vertically while it is settling towards the bottom. However, this implies that the buoyancy-corrected mass changes with the object's position. Finally, due to the fact that the cylinder is hollow, we can vary the mass in the same way as we have done for the ping-pong ball, namely by filling it with bronze particles.

All the phenomena observed for the sphere are also present for the settling cylinder (Fig. 7): We observe both the bulk oscillations and the stop-go cycles near the bottom. A few differences are clearly visible as well: First, the bulk oscillations are significantly larger in amplitude, which could be either due to the increase in contact surface or to the flatness of the bottom of the cylinder. The frequency is again independent of the mass of the object; however, it is approximately a factor 2 lower than that observed for the sphere. Although only a few oscillations are visible, they appear undamped for the higher masses but seem to be damped for the lowest mass. This is most likely due to the change in the buoyancy-corrected mass, which for this lightest case decreases from 35 to $20 \mathrm{~g}$ between impact and the first stop-go-cycle. Second, we see that the number of consecutive stop-go cycles is larger than for the sphere. We observe up to seven cycles, while for the sphere this was limited to only two or three cycles. In addition,

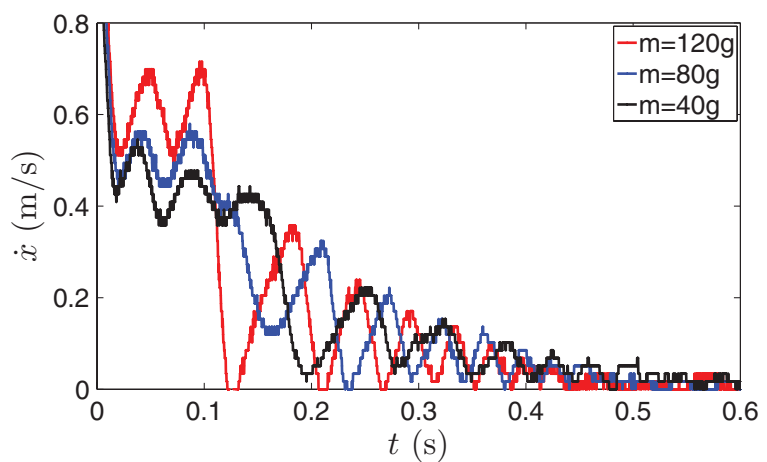

FIG. 7. (Color online) Time evolution of the velocity $\dot{x}$ of a settling cylinder in a cornstarch suspension $(\phi=0.44)$ for different cylinder masses $m_{\text {cyl }}$, varying from 40 to $120 \mathrm{~g}$. A buoyancy-corrected mass cannot be used here since it changes along the trajectory of the cylinder.

we observe that the first stop appears at a larger distance from the bottom, namely several centimeters, as compared to typically $1 \mathrm{~cm}$ for the sphere. Finally, the drop in maximum velocity between consecutive stops is smaller for the cylinder.

\section{BULK OSCILLATIONS}

When an object is settling in a fluid it can be described by the following equation:

$$
m \ddot{x}=\mu g+D(x, \dot{x}, t),
$$

in which $x(t)$ is the trajectory of the object, where $x=0$ has been chosen to coincide with the bottom of the container and $x$ increases in the downward direction. Furthermore, $g=$ $9.81 \mathrm{~m} / \mathrm{s}^{2}$ is the acceleration of gravity, $\mu$ is the previously introduced buoyancy-corrected mass, and $m=m_{\text {object }}+m_{\text {added }}$ is the sum of the mass of the object and the added mass. For a sphere, we use the traditional result for Newtonian liquids $m_{\text {added }}=0.5 \rho_{S} V_{\text {sphere }}$ with $V_{\text {sphere }}$ the volume of the sphere and $\rho_{S}$ the density of the suspension. Finally, $D$ is the drag force which, in general, is a function of the object's velocity $\dot{x}$ and position $x$. The drag force could even be a functional of $x(t)$ if the history of the objects trajectory is important (which will indeed be argued to be the case). The aim of the next two sections is to find an appropriate model for the drag force $D$.

In the present section, we will start with the bulk oscillations. We will attempt to describe this phenomenon using various models and discuss their appropriateness. As we are using a shear-thickening suspension, we start off with traditional shear-thickening models that have a monotonic stress-strain curve. We then consider several viscoelastic models, which are appropriate for the description of the position oscillations that have been observed in viscoelastic liquids. Finally, we investigate a hysteresis model based on a model proposed by Deegan [18] in the context of holes in vibrated cornstarch layers [17].

\section{A. Shear-thickening model}

As cornstarch is well known for its shear-thickening behavior, the most logical first model to try is a shear-thickening model, i.e., a model in which the viscosity increases with 


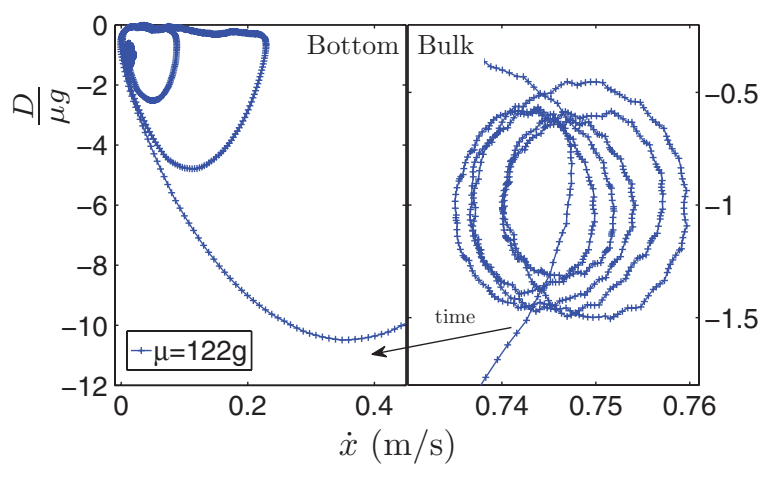

FIG. 8. (Color online) The drag $D=m \ddot{x}-\mu g$ on a ping-pong ball with buoyancy-corrected mass $\mu=122 \mathrm{~g}$ versus its velocity $\dot{x}$, calculated from the object's trajectory $x(t)$ during a settling experiment in a cornstarch suspension with $\phi=0.44$.

increasing shear rate. Or, as an alternative, one could think of a model that combines a shear rate region where the viscosity is decreasing with a region where it is increasing to model the shear-thinning-to-shear-thickening transition that has been observed in rheometer experiments in both cornstarch and other suspensions [2-7]. All these models have in common that the stress increases monotonically with the strain rate, which will lead to a monotonically increasing drag $D$ as a function of the magnitude of the objects settling velocity $\dot{x}$.

We use Eq. (1) to determine the drag force from the experimental trajectory, i.e., when we compute $D \equiv m \ddot{x}-\mu g$ for the measured acceleration $\ddot{x}$ and plot the result as a function of the velocity $\dot{x}$ we obtain Fig. 8. Clearly, during the bulk oscillations, the drag $D$ is not a monotonic function of $\dot{x}$. Therefore, we can immediately discard shear-thinning and shear-thickening models where the drag (stress) is a monotonically increasing function of the velocity (strain rate). We therefore necessarily need to turn to a model in which the object's history is important.

\section{B. Viscoelastic model}

One of the most conspicuous candidates to model the oscillatory behavior is to try a (linear) viscoelastic model. Although it is known that such models do not describe shear-thickening fluids in general, we still consider these because similar oscillations in the position and velocity of settling objects have been observed in viscoelastic liquids [24] and modeled by such models [25]. The simplest of such models is the Maxwell fluid, in which the total deformation is decomposed into an elastic term in series with a viscous term. For the elastic part, stress is proportional to strain and the proportionality constant is an elastic modulus or the viscous part stress is proportional to strain rate with (dynamic) viscosity as a proportionality constant. This translates into the following model for the drag $D$ in Eq. (1):

$$
-\dot{x}=\frac{\dot{D}}{E}+\frac{D}{\eta},
$$

where $E$ corresponds to the elastic part of the drag term and has the dimensions of a spring constant (elastic modulus times a length scale) and $\eta$ corresponds to the viscous part and has the dimensions of viscosity times length. The minus sign reflects the fact that the direction drag force will generally be opposite to the velocity of the object. The above equation can be integrated to obtain an expression for the drag in terms of $\dot{x}(t)$,

$$
D=-E \int_{t^{\prime}=0}^{t} \exp \left[-\frac{E\left(t-t^{\prime}\right)}{\eta}\right] \dot{x}\left(t^{\prime}\right) d t^{\prime} .
$$

As is shown in Sec. IV A, the equation we obtain when we insert this expression into Eq. (1) can be solved exactly using Laplace transformations, leading to

$$
\dot{x}(t)=u_{T}+e^{-\alpha t}\left[v_{0} \cos \omega t+\left(\frac{\mu g}{\omega m}+\frac{E v_{0}}{2 \omega \eta}\right) \sin \omega t\right],
$$

in which the damping rate $\alpha$, angular frequency $\omega$, the terminal velocity $u_{T}$, and $v_{0}$ are given by

$$
\begin{aligned}
\alpha & =\frac{E}{2 \eta} ; \quad \omega=\sqrt{\omega_{0}^{2}-\alpha^{2}}=\sqrt{\frac{E}{m}-\left(\frac{E}{2 \eta}\right)^{2}} ; \\
u_{T} & =\frac{\mu g}{\eta} ; \quad \text { and } \quad v_{0}=\dot{x}(0)-\frac{\mu g}{\eta} .
\end{aligned}
$$

Indeed, this solution displays oscillatory behavior as the object approaches its terminal velocity. However, the terminal velocity is directly coupled to the damping factor $\alpha=$ $E /(2 \eta)$, which in turn is coupled to the (minimum) amplitude $\mu g /(\omega m) \lesssim \mu g /\left(\omega_{0} m\right)=\mu g / \sqrt{m E} \equiv A$, which is obtained by setting $v_{0}=0$ in Eq. (4). So when we divide the amplitude over the terminal velocity we obtain

$$
\frac{A}{u_{T}}=\frac{\mu g}{\sqrt{m E}} \frac{\eta}{\mu g}=\frac{1}{2} \frac{2 \eta / E}{\sqrt{E / m}}=\frac{1}{2} \frac{\omega_{0}}{\alpha}=\pi \frac{\tau}{T},
$$

in which $\tau=\alpha^{-1}$ is the damping time and $T=2 \pi \omega_{0}^{-1}$ is the period of the oscillation. This implies that in order to have an amplitude (much) smaller than the terminal velocity, $\tau / T$ needs to be (much) smaller than 1 , i.e., the damping time should be smaller than the oscillation period. Conversely, to obtain oscillations that do not damp for several periods, one needs an amplitude which is several times larger than the terminal velocity. Therefore, we conclude that it is impossible to describe the bulk oscillations observed in cornstarch within the context of a Maxwell fluid.

It is possible to extend the Maxwell fluid to more complicated linear viscoelastic models, like the extended Maxwell fluid and the modified Kelvin-Voigt solid discussed in Sec. IV A, that contain additional dissipative elements in the hope that this would decouple terminal velocity, damping constant, and oscillation amplitude. However, as shown in Sec. IV A all of these models have a coupling similar to the one expressed in Eq. (6), which makes them unsuitable for the description of the observed phenomena. The conclusion is that the bulk oscillations in cornstarch cannot be described by a simple linear viscoelastic model like the ones we considered here. Clearly, one could think of turning to complicated viscoelastic models with multiple length and time scales. However, such an approach would not teach us much about the system. 


\section{Hysteresis model}

We therefore now turn to a model that has been proposed for the behavior of a cornstarch suspension in a different setting, namely a vertically shaken one. As mentioned in the introduction, Merkt et al. [17] have observed long-living stable holes in a vertically shaken cornstarch suspension, which they attributed tentatively to the shear-thickening properties of cornstarch. In a later paper, Deegan proposed a model to describe this behavior based on a hysteretic rheology [18]. More specifically, he proposed a coexistence of two branches in the stress versus strain rate diagram of the cornstarch suspension, the existence of which was backed up by oscillatory shear measurements in a cone-plate rheometer. This phenomenological model is able to predict the existence of growing holes in a cornstarch suspension. The historydependent coexistence of the low- and high-viscosity branch is crucial for Deegan's model and sets it aside from a model with a simplified stress-strain-rate curve with a low viscosity for low shear rates and a higher one for high shear rates. We once more stress that such a simplified model is not able to describe the observed oscillations.

We now apply this idea for our experiment of an object settling in a deep bath of cornstarch, using Eq. (1) with a hysteretic model for the drag force. More specifically,

$$
D(\dot{x})=-B(\dot{x}) \dot{x},
$$

where

$$
B(\dot{x})= \begin{cases}B_{1} & \text { when } \dot{x} \text { falls below } u_{1}, \\ B_{2} & \text { when } \dot{x} \text { rises above } u_{2} .\end{cases}
$$

Here, $u_{1}$ and $u_{2}$ (with $u_{2}>u_{1}$ ) are the turnover velocities of the system and the drag coefficients $B_{1}$ and $B_{2}$ (with $B_{2}>B_{1}$ ) are the slopes of the two branches, namely one corresponding to a low viscosity $\left(B_{1}\right)$ and the other to a high one $\left(B_{2}\right)$. Between $u_{1}$ and $u_{2}$ the system can be in either of the two branches, as illustrated in Fig. 9(a).

How this model for $D$ is able to produce oscillations in the context of Eq. (1) is illustrated schematically in Fig. 9(b). After impact, the object will be in the higher branch with drag coefficient $B_{2}$ and will decelerate until it reaches the lower boundary $u_{1}$. The system then will switch to the lower branch and its drag coefficient will decrease to $B_{1}$. As this results in a drag force smaller than the downwards acceleration of gravity, the object will accelerate again towards the terminal velocity $\dot{x}_{1}=\mu g / B_{1}$, which is the steady state of Eq. (1) when the system is in the lower branch, i.e., $D=-B_{1} \dot{x}$. Before reaching $\dot{x}_{1}$, however, the object will hit the velocity $u_{2}$, where the drag coefficient jumps to $B_{2}$. Now the object will decelerate again towards a second terminal velocity $\dot{x}_{2}=\mu g / B_{2}$ [corresponding to Eq. (1) in the higher branch $\left.D=-B_{2} \dot{x}\right]$, but before arriving there, $u_{1}$ will be reached again. This restarts the cycle that will now repeat over and over again.

To relate $B_{1}$ and $B_{2}$ to experimental observables, we solve Eq. (1) for $\dot{x}$ in each of the two branches, starting at an arbitrary time $t=t_{0}$ at one of the boundaries $u_{1}$ or $u_{2}$ of the hysteresis loop. This leads to

$$
\dot{x}=\dot{x}_{i}+\left(u_{i}-\dot{x}_{i}\right) \exp \left[\frac{-\mu B_{i}}{m}\left(t-t_{0}\right)\right],
$$

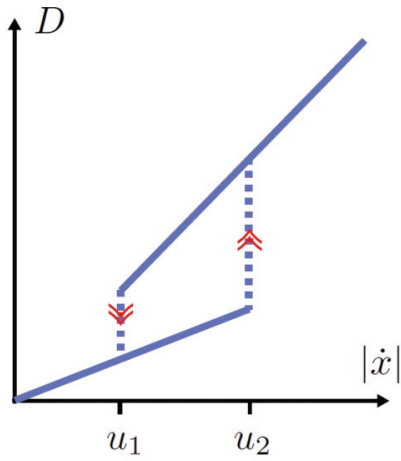

(a)

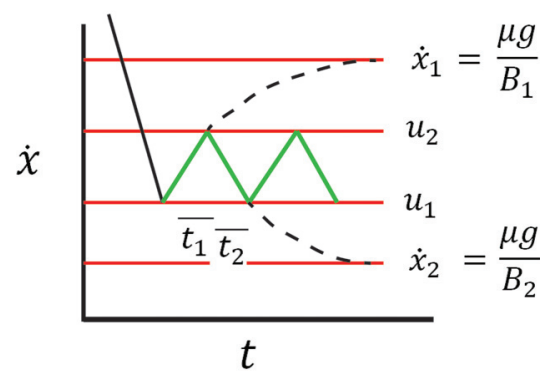

(b)

FIG. 9. (Color online) (a) Schematic of the drag force $D$ defined by Eq. (7) as a function of the velocity $\dot{x}$, with the hysteresis loop between $\dot{x}=u_{1}$ and $\dot{x}=u_{2}$. (b) Schematic of the oscillatory solution of Eq. (1) using the drag force of (a): For suitable values of $B_{1}, B_{2}, u_{1}$, and $u_{2}$ the system alternately switches from the low to the high branch in the hysteresis loop and back. In these schematics, all quantities are in arbitrary units.

for $i=1,2$. Here, $\dot{x}_{i} \equiv \mu g / B_{i}$ are the terminal velocities introduced above.

Now we linearize this equation in $t$ between $u_{1}$ and $u_{2}$. This leads to the following expression for $B_{1}$ :

$$
B_{1}=\left(\frac{-u_{2}+u_{1}}{\Delta t_{1}} \frac{m}{\mu}+g\right) \frac{1}{u_{1}}
$$

and similarly for $B_{2}$ with the indices 1 and 2 interchanged. Here, $\Delta t_{1}$ and $\Delta t_{2}$ are the time intervals it takes for the object to accelerate or decelerate from the one switching velocity to the other. By determining $\Delta t_{1}, \Delta t_{2}, u_{1}$, and $u_{2}$ from our experiments we can now calculate $B_{1}$ and $B_{2}$.

The next step is to compare the model to the experiments. First, we assumed that the drag coefficients $B_{1}$ and $B_{2}$ are determined by fluid properties, i.e., that they are independent of the object mass or velocity. We thus calculated the drag coefficients for the experiment with the highest mass and applied them to the other masses. Here, we do have to adjust the turnover velocities $u_{1}$ and $u_{2}$ to obtain an oscillation between the observed velocity boundaries. The result is shown as the solid lines in Fig. 10(a). We apply the model after all impact related effects have disappeared and clear fluctuations around a terminal velocity are visible. For the four measurements between $\mu=62$ and $\mu=132 \mathrm{~g}$ in Fig. 10(a), we see that the model fits nicely for the heaviest balls, and quite well for the lighter balls, from which we conclude that the assumption of constant $B_{1}$ and $B_{2}$ is reasonable. This can also be checked 


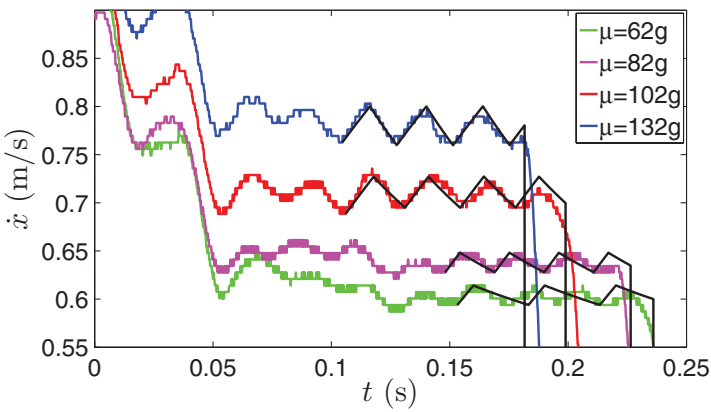

(a)

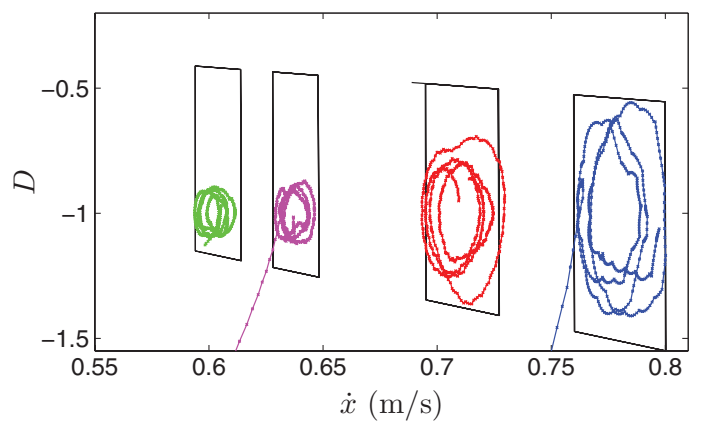

(b)

FIG. 10. (Color online) Comparison of the experimental results to the hysteresis model. (a) In the region where bulk oscillations are observed, the velocity of the sphere is plotted versus time for four different buoyancy-corrected masses $(\mu=62,82,102$, and $132 \mathrm{~g})$ for both the experiment (colored symbols) and the model (black lines), where the values for $B_{1}$ and $B_{2}$ have been obtained from the experiment with the highest mass. (b) The corresponding drag $D=m \ddot{x}-\mu g$ versus velocity $\dot{x}$ plots also for both the experiment (colored symbols) and the model (black lines).

by calculating $B_{1}$ and $B_{2}$ for every experiment separately, the results of which are shown in Fig. 11 . We observe that both $B_{1}$ and $B_{2}$ vary only slightly for all masses.

For further comparison, we plot the hysteresis loop in a drag versus velocity plot for both the experiment and the model in Fig. 10(b). It is clear that the modeled loop is a very simplified representation of the actual loop, and that, especially for the

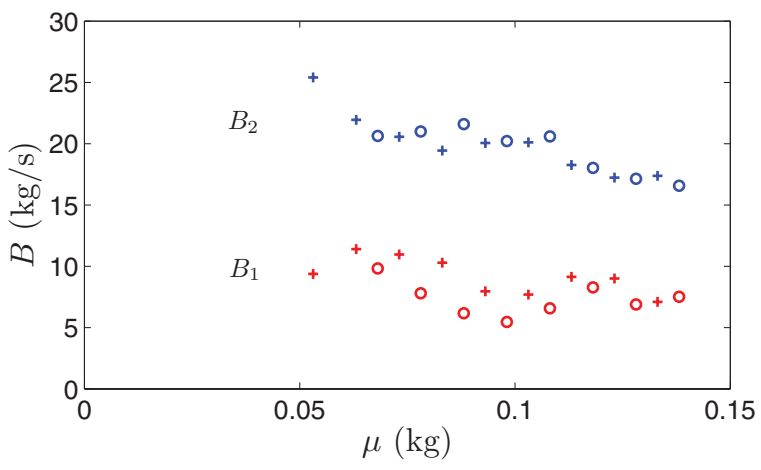

FIG. 11. (Color online) The drag coefficients $B_{1}$ and $B_{2}$ as a function of buoyancy-corrected mass $\mu$, now calculated from the experiment using Eq. (10) separately for each value of $\mu$. Below $\mu=$ $62 \mathrm{~g}$ no bulk oscillations could be discerned. The different symbols correspond to two different series of experiments.

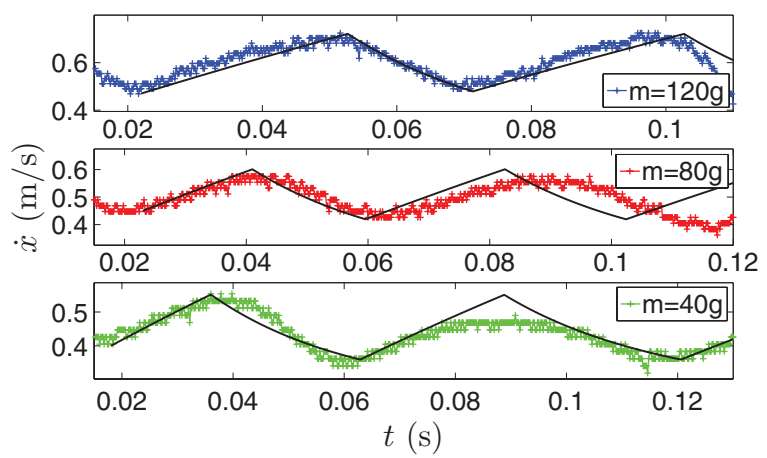

FIG. 12. (Color online) Comparison of the experimental results to the hysteresis model for a cylindrical object in the region where bulk oscillations are observed. The velocity of the cylinder is plotted versus time for three different masses $\left(m_{\text {cyl }}=40,80\right.$, and $120 \mathrm{~g}$ ) for both the experiment (colored symbols) and the model (black lines), where the values for $B_{1}$ and $B_{2}$ have been obtained from the experiment with the highest mass.

lower masses, the variations in the observed accelerations are considerably smaller than those of the model.

We compared the experiments with a spherical object to those with a cylinder (discussed in Sec. IIIE). The cylinder diameter is $1.5 \mathrm{~cm}$, which is smaller than the ball, and also we now have a flat bottom instead of a round one. Due to the length of the cylinder we expect more interaction between the liquid and the object, but we also have to keep in mind that we now have a buoyancy that increases during sedimentation. Again, we calculate $B_{1}$ and $B_{2}$ from the measurements with the heaviest mass, where the effect is most pronounced, and adjust $u_{1}$ and $u_{2}$ for each mass. The results are shown in Fig. 12 where we see that (as expected) the fit is very nice for the highest mass and that there are larger discrepancies for the lower masses. The used values for $B_{1}$ and $B_{2}$ for the cylinder are respectively approximately a factor 2 lower and higher compared to the values used for the sphere, 3 and 35 for the cylinder vs. 7 and 18 for the sphere.

Concluding this section, we found that traditional shearthinning and shear-thickening models are not able to describe the bulk oscillations due to the presence of history dependence (hysteresis) in the experiments. In addition, we found that simple linear viscoelastic models fail to describe the observed oscillations due to an intrinsic coupling between the terminal velocity and the oscillation amplitude in these models that is inconsistent with the experiments. The best candidate is a description in terms of a hysteretic drag term inspired by the work of Robert Deegan [18]. One could say that the major drawback of the model is that it is entirely phenomenological, i.e., a physical mechanism to relate its parameters to the physics of the system is still lacking.

The experiments point to a physical mechanism where the origin of the oscillations is a modulation on top of the terminal velocity, due to periodically changing properties in the liquid. More specifically, one could imagine a jammed region around the object which grows when it moves fast, through which the drag increases causing the object to decelerate. The object would than obtain a lower velocity which would allow relaxation and shrinking of the jammed layer, i.e., a decrease of the drag and an acceleration of the object. For the bulk 
oscillations there are many open questions to propose such a model, but for the stop-go cycles at the bottom the formulation of such a model is feasible, as we will show in the next section.

\section{STOP-GO CYCLES}

As was shown in Sec. III, we always observe stop-go cycles near the bottom at cornstarch concentrations higher than $\phi=$ 0.38 . Here, the object suddenly stops one or a few centimeters above the bottom of the container. It then accelerates again and comes to another abrupt stop a little closer to the bottom. This cycle repeats itself several times.

As explained in Ref. [21], we interpret these stop-go cycles as follows: While the object is moving down, the cornstarch below it is slowly being compressed such that at a certain moment a jammed network of particles forms between the object and the container bottom. This jammed layer is responsible for the large force that brings the object to a full stop. Stresses build up in the network and therefore also within the interstitial fluid, which triggers a Darcy's flow in the porous medium formed by the cornstarch grains, allowing the network to relax through (small) particle rearrangements. This causes the jammed region to unjam and the object will start moving again. Such hardening of a cornstarch suspension has also been reported in Ref. [22], where a ball was pushed towards the bottom, leaving an indent on a clay layer on the bottom. This was attributed to forces being transmitted through a hardened layer beneath the ball.

In Fig. 13 we compare the stop-go cycles for the settling ping-pong ball (which we previously presented in Ref. [21]) with those for the settling cylinder, both for three different values of the buoyancy-corrected mass $\mu$. Clearly, for the cylinder there are more stop-go cycles than there are for the ping-pong ball. To further quantify the stop-go cycles, we measure the distance from the bottom at which the object stops for the first time $\left(\left|x_{0}\right|\right)$, Fig. 14(a), the maximum velocity it reaches after the first stop ( $\left.\dot{x}_{\max }\right)$, Fig. 14(b), and the time it needs to reach this velocity $\left(t_{\text {acc }}\right)$, Fig. 14(c), for both the ping-pong ball and the hollow cylinder. Although in general the cylinder has a variable buoyancy-corrected mass during its trajectory, due to the proximity of the bottom we could define a meaningful buoyancy-corrected mass here. Namely, we choose the cylinder mass minus the buoyancy the cylinder would experience when it would be at the bottom.

We see that the cylinder stops several centimeters above the bottom, i.e., several centimeters above the first stop of the ping-pong ball. Furthermore, in comparison with the ball, it reaches higher velocities after the first jump but requires approximately the same time to get there. The earlier stop can be explained by a relatively larger jammed region due to the flat bottom of the cylinder, which might also give a more confined region as compared to that below the curved surface of the ball. The fact that the cylinder accelerates to a higher velocity is presumably due to the first stop being at a larger distance from the bottom, such that it takes longer for the material to jam again.

We model this process by coupling the equation of motion Eq. (1) to an equation for an order parameter which indicates whether the cornstarch suspension layer between the object and the bottom is jammed. For this, we will use the local
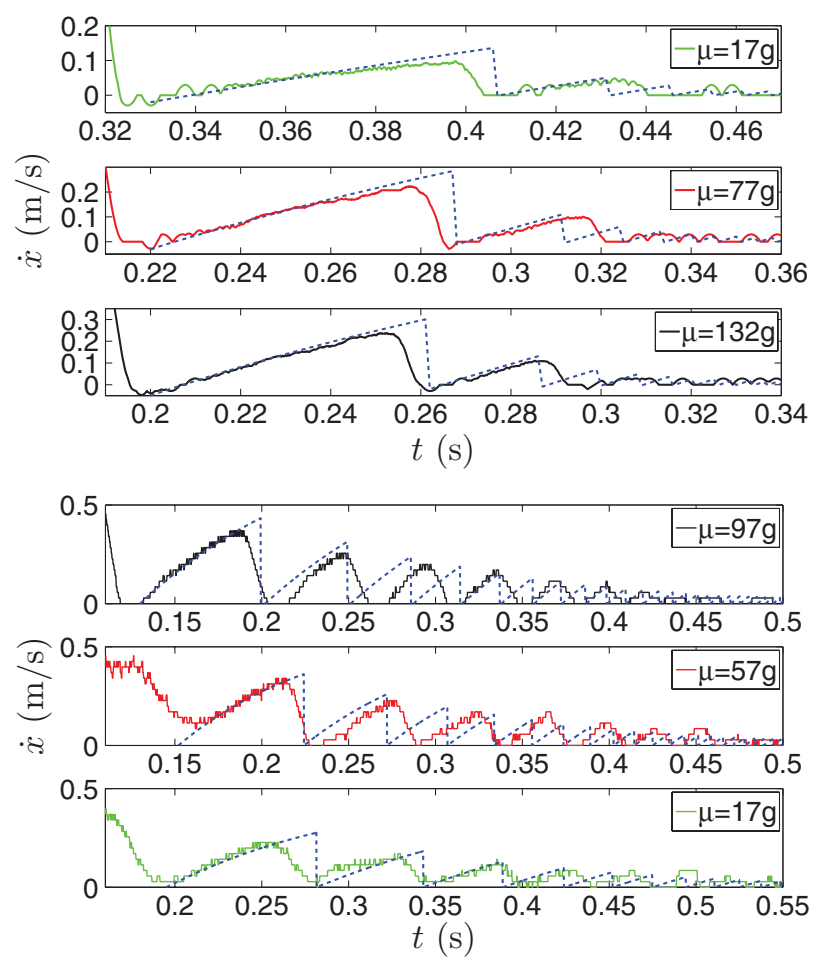

FIG. 13. (Color online) Time evolution of the velocity during the stop-go cycles for the settling ping-pong ball for three different (buoyancy-corrected) masses, $\mu=17,77$, and $132 \mathrm{~g}$, and for the settling cylinder, also for three different buoyancy-corrected masses, $\mu=97,57$, and $17 \mathrm{~g}$. The noisy lines represent the experimental results and the dashed blue lines correspond to the model of Eq. (11).

particle volume fraction $\phi$. More specifically, we assume that when $\phi$ exceeds a critical value $\phi_{\mathrm{cr}}$ the layer is jammed and the drag force $D$ is assumed to become infinitely large until the sphere comes to a standstill. This leads to the following modification of the freefall equation:

$$
\left\{\begin{aligned}
m \ddot{x} & =\mu g+D & \text { when } \phi<\phi_{\text {cr }} \\
\dot{x} & =0 & \text { when } \phi \geqslant \phi_{\text {cr }}
\end{aligned}\right\} .
$$

Due to the comparatively low velocities in this regime compared to those of the bulk-oscillations regime, we can take $D=-B \dot{x}$. Since the cornstarch layer below the object jams through compression, the equation for the time rate of change of the packing fraction $\phi$ should contain a term that increases $\phi$ proportional to the compression rate $-\dot{x} / x$ of this layer. In addition, there should be a term that decreases $\phi$ through a relaxation process towards its equilibrium value $\phi_{\mathrm{eq}}$, which is taken to be equal to the value that it has in the quiescent cornstarch suspension. This yields

$$
\dot{\phi}=-c \frac{\dot{x}}{x}-\kappa\left(\phi-\phi_{\mathrm{eq}}\right),
$$

in which $c$ and $\kappa$ are the proportionality constants of the compression and relaxation processes, respectively. Note that $c$ is dimensionless, while $\kappa$ is a relaxation time.

The critical packing fraction $\phi_{\mathrm{cr}}$ is the value at which the cornstarch suspension dynamically jams. It must lie between the static, sedimented (0.44) and the maximally compacted (0.57) value [12]. In our laboratory we tried to create the 

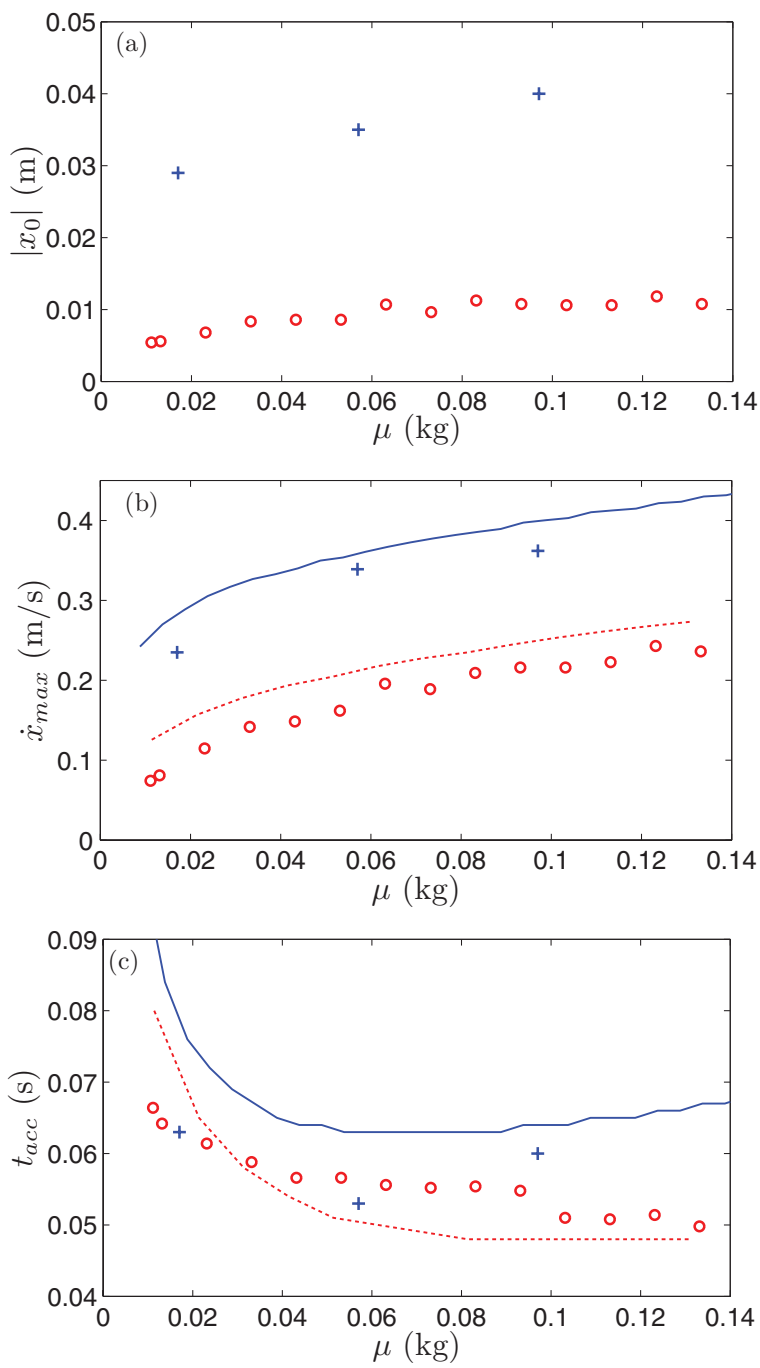

FIG. 14. (Color online) Three quantities that characterize the first stop-go cycle as a function of buoyancy-corrected mass for the cylinder (blue crosses) and pingpong ball (red circles): (a) Distance $x_{0}$ to the bottom at which the first stop takes place. (b) The maximum velocity reached in the relaxation period after the first stop. (c) The time needed to reach the maximum velocity after the first stop. The solid lines in (b) and (c) represent the results obtained with the model of Eq. (11).

densest, still flowing cornstarch suspension from which we estimate that $\phi_{\text {cr }}=0.46$ at most. As a result, $\phi$ only varies marginally during the process, in agreement with recent research where, during jamming of a cornstarch suspension in a Couette cell, no density differences were measured within experimental accuracy (0.01) of the MRI device used [15].

To obtain the time evolution of $x(t), \dot{x}(t)$, and $\phi(t)$ this set of equations needs to be solved with the initial conditions the system has reached just after the first stop: $x(0)=-x_{0}$, the position of the object at the first stop (with $x_{0}>0$ ), $\dot{x}(0)=0$ and $\phi(0)=\phi_{\mathrm{cr}}$. This immediately points to a convenient way of nondimensionalizing the equations, namely by using $x_{0}$ and $\sqrt{x_{0} / g}$ as the appropriate length and time scales. With the nondimensional variables $\tilde{x} \equiv x / x_{0}, \widetilde{t} \equiv t / \sqrt{x_{0} / g}$, and $\delta \widetilde{\varphi} \equiv$ $\left[\phi(t)-\phi_{\mathrm{eq}}\right] /\left(\phi_{\mathrm{cr}}-\phi_{\mathrm{eq}}\right)$, the set of equations becomes

$$
\begin{array}{rlrl}
\dot{x} & =\mu / m-\widetilde{B} \tilde{x} \quad \text { when } \quad \delta \tilde{\varphi}<1, \\
\tilde{x} & =0 & \text { when } \quad \delta \tilde{\varphi} \geqslant 1, \\
\delta \tilde{\varphi} & =-\tilde{c} \frac{\tilde{x}}{\tilde{x}}-\tilde{\kappa} \delta \tilde{\varphi}, &
\end{array}
$$

with initial conditions $\tilde{x}(0)=-1, \dot{\widetilde{x}}(0)=0$, and $\delta \widetilde{\varphi}(0)=1$. The dimensionless model parameters are now $\mu / m, \widetilde{B} \equiv$ $(B / m) \sqrt{x_{0} / g}, \widetilde{c}=c /\left(\phi_{\mathrm{cr}}-\phi_{\mathrm{eq}}\right)$, and $\widetilde{\kappa}=\kappa \sqrt{x_{0} / g}$, which is the ratio between the gravitational time scale and the relaxation time scale.

What can we say about the parameters in these equations with respect to our experiments? First, $\mu / m$ is expected to be of order unity. Second, for most of our experiments the acceleration phase in a cycle appears to be dominated by gravity, such that the second term in Eq. (11) is much smaller than the first, implying that $\widetilde{B} \ll 1$. Third, since $x$ and changes in $x$ during a single cycle are of the same order, we expect by neglecting the last term in the last equation for that $\delta \widetilde{\varphi} \sim \widetilde{c}$. Since, on the other hand, $\delta \widetilde{\varphi} \approx 1$, because the compression is expected to change $\phi$ from its bulk value $\phi_{\text {eq }}$ to the critical value $\phi_{\mathrm{cr}}$, we expect $\widetilde{c}$ to be of order 1 .

If we fit our model to the experimental data of the ping-pong ball we find a best fit for $c=0.025$, which with $\phi_{\mathrm{cr}}-\phi_{\mathrm{eq}} \approx$ 0.02 implies that $\widetilde{c} \approx 1.3$, in agreement with our expectation.

Finally, for the last parameter of our model, $\kappa$, some more extensive analysis is necessary. To this end, let us note that the last equation of Eq. (13) can be immediately solved implicitly using the integrating factor method. We then find that $\exp (\widetilde{\kappa} t)$ is the integrating factor for this equation leading to

$$
\delta \widetilde{\varphi}(\tilde{t})=e^{-\widetilde{\kappa} \widetilde{t}}\left[1+\widetilde{c} \int_{\widetilde{t}^{\prime}=0}^{\widetilde{t}} \frac{\tilde{x}\left(\tilde{t}^{\prime}\right)}{\widetilde{x}\left(\widetilde{t}^{\prime}\right)} e^{\widetilde{\kappa} \tau^{\prime}} d \widetilde{t}^{\prime}\right],
$$

where we have used the initial condition $\delta \tilde{\varphi}(0)=1$. Of course, the solution $\tilde{x}(\tilde{t})$ of the first equation still needs to be inserted in this equation. Since $\widetilde{B} \ll 1$, we now neglect the second term in the first equation of Eq. (13) we simply find that $\tilde{x}(\tilde{t})=$ $-1+\frac{1}{2}(\mu / m) \tilde{t}^{2}$. Using this approximation with $\mu / m=1$ and setting $\widetilde{c}=1$ in Eq. (14), we can calculate the duration $\Delta \widetilde{t}$ of and the traveled distance $\Delta \tilde{x}$ during the cycle.

These quantities are plotted as a function of the relaxation parameter $\widetilde{\kappa}$ in Fig. 15 . For very small values of $\widetilde{\kappa}(<0.1)$ we have very small durations and traveled distance. This happens because now the relaxation time scale is much larger than the gravitational time scale, which implies that as soon as the jammed layer starts to relax, the system quickly reaccelerates and jams again. $\delta \widetilde{\phi}$ will never move far from 1, i.e., $\phi$ will always be close to $\phi_{\mathrm{cr}}$. Here the object effectively gets stuck inside the cornstarch suspension (at the observable, gravitational time scales). On the other hand, for very large values of $\widetilde{\kappa}(>10)$ the opposite happens: we have a duration close to $\sqrt{2}$ corresponding to a traveled distance of $\Delta \widetilde{x} \approx 1$, which means that in this single cycle the object moves all the way to the bottom and only jams when it very quickly squeezes the very last thin layer of suspension. Here, the relaxation time scale is much smaller than the gravitational time scale, which implies that the system very quickly relaxes to the quiescent state $\phi \approx \phi_{\text {eq }}$ and the gravitational acceleration is not fast 


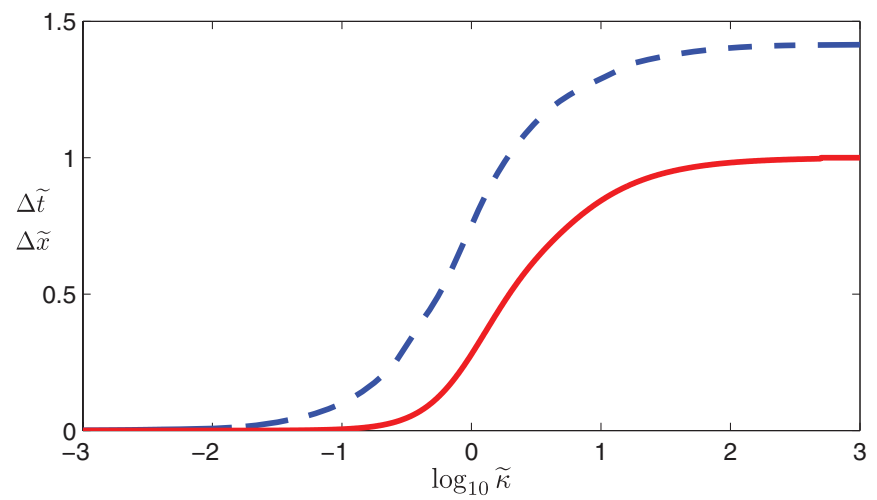

FIG. 15. (Color online) The duration $\Delta \tilde{t}$ (dashed blue curve) of and the traveled distance $\Delta \tilde{x}$ (solid red curve) during a stop-go cycle as a function of the logarithm of the relaxation parameter $\widetilde{\kappa}$. Modeled with Eq. (14), using $\widetilde{B} \ll 1$ and $c=1$.

enough to jam the material. Here the object effectively never jams and never goes into a stop-go cycle.

Now, when we fit our model to the experimental data of the ping-pong ball we find a best fit for $\kappa \approx 40 \mathrm{~s}^{-1}$, which corresponds to a relaxation time scale of $1 / \kappa \approx 0.025 \mathrm{~s}$. Since for $x_{0} \approx 1 \mathrm{~cm}$ the gravitational time scale is $\sqrt{x_{0} / g} \approx 0.032 \mathrm{~s}$, this leads to $\widetilde{\kappa} \approx 1.3$. In Ref. [21] we found the value of $\kappa \approx 40 \mathrm{~s}^{-1}$ to be connected to a Darcy's flow on a scale of 100 cornstarch particle diameters, which is consistent with mesoscopic length scales that have been found in these suspensions [14]. With this model we also understand why we do not see the stop-go cycles for lower packing fractions, as $\kappa$ will be larger and $c$ will be smaller. As a result, the effect will move closer to the bottom, such that it is not measurable within our experimental resolution.

Above, and in Ref. [21], we observed that the model works quite well for the first stop-go cycle of the spherical object but fails for the subsequent cycles which we attributed to the curved surface of the sphere. However, we expect the model to work better for a cylinder, which has a flat bottom and, therefore, the jammed cornstarch suspension layer is expected to be closer to the modeled cylindrical shape than for the sphere. When we compare the model to the experiments, see Fig. 13, we indeed see that now the second stop-go cycle also matches the experiment quite well, and the experimentally observed number of cycles comes closer to the number we see in the model. Another important point to make is that we kept the same value for $\kappa=40 \mathrm{~s}^{-1}$ as for modeling the ping-pong ball, which indeed should be just dependent on the suspension. We did increase the compression factor from 0.025 for the sphere to 0.07 for the cylinder, which also stands to reason given the different geometry.

To quantify this difference between the ball and the cylinder, we plot the maximal velocities in the successive stop-go cycles in Fig. 16, normalized by the average bulk velocity, for both the experiments and the model. Clearly, the experiments of the ball and those of the cylinder follow two different paths: In the case of the ping-pong ball the cycles damp out very fast, whereas the cylinder has a more gradual decrease in maximal velocities. The model follows both sets quite well, but overpredicts the

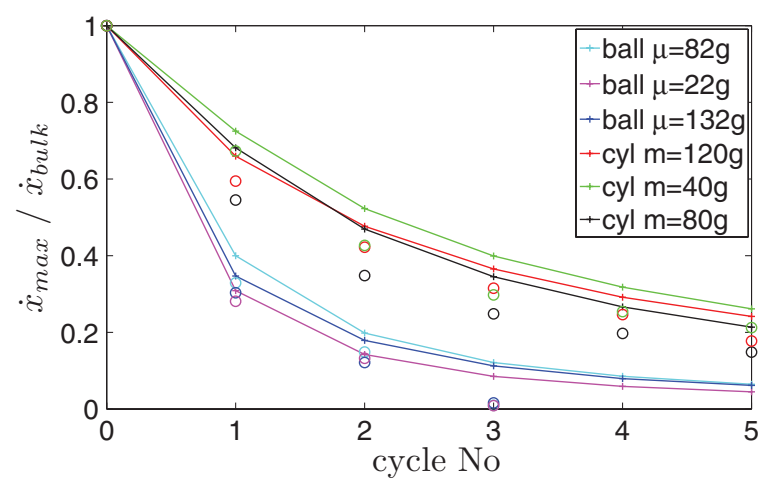

FIG. 16. (Color online) The maximum velocities reached during the stop-go cycles in the experiments (circles) and the model (lines) per jump, normalized by the mean bulk velocity, positioned at cycle 0 .

number of cycles for the ball to a larger extent than for the cylinder, consistent with the above arguments.

\section{OTHER SUSPENSIONS}

It is known that different suspensions can behave quite differently, which is presumably connected to particle shape and size distribution. An example of this is their behavior when shaken vertically [17-20], where for comparable solid fractions one may, e.g., observe stable holes, growing or even splitting holes, or closing holes, depending on the suspension that was used. We therefore repeated our settling experiments in a variety of other suspensions. Although dense suspensions of particles are all shear thickening [2], both the bulk oscillations and the stop-go cycles observed in cornstarch are absent in the other suspensions we studied. This can be seen in Fig. 17, where we present settling velocities for suspensions containing quartz flour, glass beads, polystyrene beads, and compare these with a cornstarch suspension.

More specifically, as alternatives to cornstarch, we used monodisperse spherical particles made of polystyrene with a diameter of $20 \mu \mathrm{m}$, polydisperse glass spheres with diameters between 0 and $20 \mu \mathrm{m}$, and quartz flour of $0-50 \mu \mathrm{m}$, in which the particles have edgy, irregular shapes.

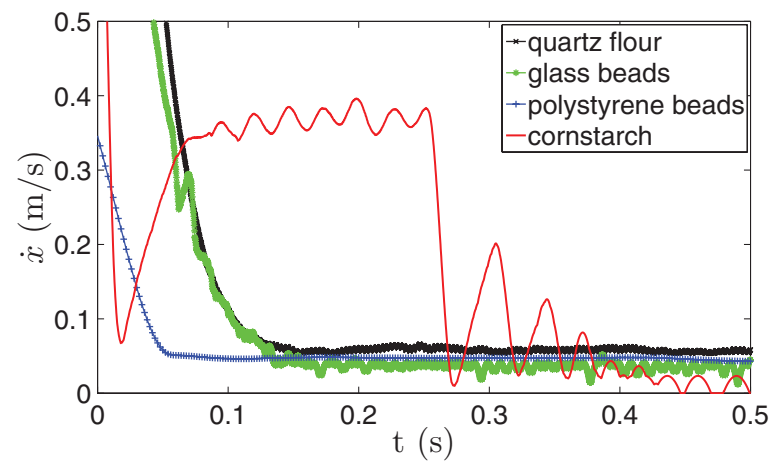

FIG. 17. (Color online) Time evolution of the velocity of a steel sphere with a diameter of $1.6 \mathrm{~cm}$ in three different suspensions: quartz flower, polystyrene beads, glass beads, and cornstarch. Clearly, both the bulk oscillations and the stop-go cycles are only observable in the cornstarch suspension. 
For the glass and quartz flour particles, only thin layers were used without full density matching and with a very high solid fraction. In these thin layers, we hoped to encounter stop-go cycles but these were not observable. It is possible that the absence of the stop-go cycles is caused by the size distribution of these particles, because in both cases there is a large amount of small particles that can get between the fewer larger ones. This causes that no sudden rearrangements of particles can happen, which we believe to be the cause of the phenomena we see. For the monodisperse spherical polystyrene beads ( $d=20 \mu \mathrm{m}, \phi \sim 0.6$ ) we did use a deep bath of suspension. However, also in this case no bulk oscillations or stop-go cycles were observed. Another difference is that the instant velocity drop after impact on a cornstarch suspension [27] is far less abrupt for the other suspensions we used, which suggests that a much smaller jammed region is created below the impacting object compared to cornstarch.

The remaining questions is what sets aside cornstarch to these particles. If the origin is geometrical, it is most likely a combination of size, size distribution, and shape. It would be interesting if there would exist an alternative to cornstarch, i.e., a rather monodisperse sample of edgy, cubelike particles with diameters of $20 \mu \mathrm{m}$ or somewhat larger, that could be produced in large enough quantities to perform settling experiments. To our knowledge such an alternative is not available.

\section{CONCLUSIONS}

In conclusion, we presented experiments of objects settling into a dense bath of a cornstarch suspension, which revealed pronounced non-Newtonian behavior: Instead of reaching a terminal velocity and monotonously stopping at the bottom, the object's velocity oscillates within the bulk and goes through a series of stop-go cycles at the bottom. These effects are not observed in a wide range of other dense suspensions, leading us to believe that cornstarch particles have some unique property. We were not able to pinpoint this property, but suggest that it may be connected to the particular shape and size distribution of the particles.

We studied the influence of several parameters on the observed phenomena and found that both disappear if the solid fraction of cornstarch particles is below $\phi \approx 0.38$. This suggests that contact forces must play an important role in creating the observed behavior. If the density ratio between the object and the cornstarch suspension (which always is larger than 1) becomes low, the bulk oscillations disappear, but the stop-go cycles are still clearly observable. Other parameters that were studied are the object shape and the container size.

We discussed several models in the context of the bulk oscillations and concluded that common shear-thickening and linear viscoelastic models fail to account for the observed phenomena. A hysteretic drag model captures the basic phenomenology in the bulk. However, the model is entirely phenomenological, and a link between the model parameters and the physical properties of the system still needs to be established.

We proposed a jamming model that describes the stop-go cycles near the bottom and discuss its properties and its plausibility. The model is capable of describing both our experiments with spheres and cylinders of variable mass and works particularly well for the experiments done with a cylinder. This stands to reason because the geometry of the cylinder is closest to the geometry assumed in the model.

\section{ACKNOWLEDGMENTS}

The authors thank Matthias van de Raa for his work in the quasi-two-dimensional setup. The work is part of the research program of FOM, which is financially supported by NWO; S.v.K. acknowledges financial support.

\section{APPENDIX: LINEAR VISCOELASTIC MODELS}

In this Appendix we will first discuss a model for the drag a sphere experiences based on the Maxwell fluid rheology. Subsequently, we will solve the equation of motion for a settling sphere [Eq. (1)] in such a fluid using Laplace transformations, and, finally, we will discuss extensions to the Maxwell fluid, namely the extended Maxwell model and the modified Kelvin-Voigt solid, that contain additional dissipative elements.

Linear viscoelastic models for the drag force $D$ (just like the constitutive stress-strain (rate) relations for a viscoelastic fluid) are equations composed of elastic and viscous terms which provide a relation between drag and displacement for the first and drag and velocity for the second or

$$
\dot{D}=-E \dot{x} ; \quad D=-\eta \dot{x},
$$

in which we have taken the time derivative of the first relation for practical reasons. The proportionality constant $E$ has the dimensions of a spring constant and $\eta$ has the dimensions of viscosity times length.

A Maxwell fluid consists of an elastic and a viscous term in series [Fig. 18(a)], such that $\dot{x}$ is the sum of an elastic and a viscous part that both are subject to the same force such that

$$
-\dot{x}=\frac{\dot{D}}{E}+\frac{D}{\eta} .
$$

Writing $D=\int_{0}^{t} \psi\left(t-t^{\prime}\right) \dot{x}\left(t^{\prime}\right) d t^{\prime}$ and inserting this into Eq. (A2) impliess that $\psi(t)$ should be the solution of that equation with $\dot{x}=\delta(t)$, the Dirac $\delta$ function. This can be solved by first finding the solution to the homogeneous problem [inserting $\dot{x} \equiv 0$ in Eq. (A2)] and subsequently integrating the full equation [with $\dot{x}=\delta(t)$ ] over a short interval around

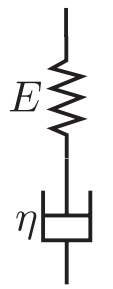

(a)

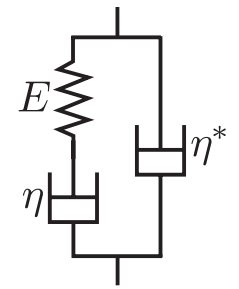

(b)

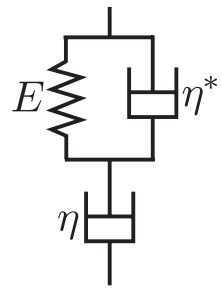

(c)
FIG. 18. Schematics of the three linear viscoelastic models discussed in this Appendix: (a) Maxwell fluid, (b) extended Maxwell fluid, and (c) modified Kelvin-Voigt solid. 
$t=0$. This yields

$$
\psi(t)=-E \exp \left[-\frac{E t}{\eta}\right],
$$

leading to the drag of Eq. (3) and by insertion into the equation of motion Eq. (1) we obtain

$$
m \ddot{x}=\mu g-E \int_{t^{\prime}=0}^{t} \exp \left[-\frac{E\left(t-t^{\prime}\right)}{\eta}\right] \dot{x}\left(t^{\prime}\right) d t^{\prime} .
$$

Introducing the Laplace transforms of the velocity $u=\dot{x}$ and the function $\psi$, namely $\widetilde{u}(s) \equiv \int_{0}^{\infty} \exp (-s t) \dot{x}(t) d t$ and $\widetilde{\psi}(s) \equiv \int_{0}^{\infty} \exp (-s t) \psi(t) d t$, respectively, we obtain by transforming Eq. (A4) using standard Laplace techniques

$$
m s \tilde{u}(s)-m u(0)=\frac{\mu g}{s}+\tilde{\psi}(s) \tilde{u}(s) .
$$

Also, we obtain from Eq. (A3) that $\widetilde{\psi}(s)=-E /(s+E / \eta)$ with which

$$
\left(m s+\frac{E}{s+E / \eta}\right) \widetilde{u}(s)=\frac{\mu g}{s}+m u(0) .
$$

The limit $t \rightarrow \infty$ corresponds to the limit $s \downarrow 0$ of the Laplace transform, which when applied to Eq. (A6) leads to $\lim _{s \downarrow 0} \widetilde{u}(s)=\mu g /(\eta s)$ or $\lim _{t \rightarrow \infty} u(t)=\mu g / \eta$. Therefore, there is a terminal velocity $u_{T}=\mu g / \eta$. Subtracting this terminal velocity by introducing a new variable $\widetilde{v}=\widetilde{u}-\mu g /(\eta s)$ [or, equivalently, $v(t)=u(t)-\mu g / \eta$ ] we obtain after some algebraical manipulation of Eq. (A6)

$$
\widetilde{v}(s)=v_{0} \frac{s+\alpha}{(s+\alpha)^{2}+\omega^{2}}+\left(\frac{\mu g}{\omega m}+\frac{E v_{0}}{2 \omega \eta}\right) \frac{\omega}{(s+\alpha)^{2}+\omega^{2}},
$$

with $\alpha=E /(2 \eta), \omega=\sqrt{E / m-\alpha^{2}}$, and $v_{0}=u(0)-\mu g / \eta$. The (standard) inverse transform of this equation directly leads to Eq. (4),

$$
\dot{x}(t)=u_{T}+e^{-\alpha t}\left[v_{0} \cos \omega t+\left(\frac{\mu g}{\omega m}+\frac{E v_{0}}{2 \omega \eta}\right) \sin \omega t\right] .
$$

The slightly more complicated extended Maxwell fluid [Fig. 18(b)] and modified Kelvin-Voigt solid [Fig. 18(c)] are defined by

$$
\begin{aligned}
D & =D_{L}+D_{R} \\
-\dot{x} & =\dot{D}_{R} / E+D_{R} / \eta=D_{L} / \eta^{*},
\end{aligned}
$$

for the extended Maxwell fluid, and

$$
\begin{aligned}
D & =D_{L}+D_{R} \\
-\dot{x} & =D / \eta+D_{R} / \eta^{*}=D / \eta+\dot{D}_{L} / E,
\end{aligned}
$$

for the modified Kelvin-Voigt solid. Along a similar path leading to Eq. (A3), this leads to the following equation for the extended Maxwell fluid (eM) kernel $\psi_{\mathrm{eM}}(t)$

$$
\psi_{\mathrm{eM}}(t)=-\left[E \exp (-(E / \eta) t)+\eta^{*} \delta(t)\right],
$$

which leads directly to its Laplace transform

$$
\widetilde{\psi}_{\mathrm{eM}}(s)=-\left[\frac{E}{s+E / \eta}+\eta^{*}\right] \text {. }
$$

For the modified Kelvin-Voigt solid (mKV) the situation is slightly more complicated because the analysis leads to an integral equation for $D$ and $\dot{x}$ that appears hard to solve for $D$,

$$
\begin{gathered}
\int_{0}^{t}\left[\left(1+\eta^{*} / \eta\right) \delta\left(t-t^{\prime}\right)+E / \eta\right] D\left(t^{\prime}\right) d t^{\prime} \\
=-\int_{0}^{t}\left[E+\eta^{*} \delta\left(t-t^{\prime}\right)\right] \dot{x}\left(t^{\prime}\right) d t^{\prime} .
\end{gathered}
$$

However, we only are interested in the Laplace transform $\widetilde{\psi}_{\mathrm{mKV}}(s)$ which is readily obtained from the Laplace transform of the above equation realizing that $\widetilde{\psi}_{\mathrm{mKV}}(s) \equiv \widetilde{D}(s) / \widetilde{u}(s)$,

$$
\widetilde{\psi}_{\mathrm{mKV}}(s)=-\frac{E / s+\eta^{*}}{\left(1+\eta^{*} / \eta\right)+E /(\eta s)} .
$$

Actually, both kernels are equivalent, which can be seen by introducing a set of new parameters, namely for eM: $\bar{E} \equiv E\left(1+\eta^{*} / \eta\right), \bar{\eta} \equiv \eta\left(1+\eta^{*} / \eta\right)$, and $\bar{\eta}^{*} \equiv \eta^{*}$, and for $\mathrm{mKV}: \bar{E} \equiv E /\left(1+\eta^{*} / \eta\right), \bar{\eta} \equiv \eta$, and $\bar{\eta}^{*}=\eta^{*} /\left(1+\eta^{*} / \eta\right)$. With this, both kernels become

$$
\widetilde{\psi}(s)=-\frac{\bar{E}+\bar{\eta}^{*} s}{s+\bar{E} / \bar{\eta}} .
$$

Inserting this $\psi(s)$ into the equation of motion Eq. (A5) gives

$$
\left(m s+\frac{\bar{E}+\bar{\eta}^{*} s}{s+\bar{E} / \bar{\eta}}\right) \widetilde{u}(s)=\frac{\mu g}{s}+m u(0) .
$$

Again, the limit $t \rightarrow \infty$ teaches us that there is a terminal velocity $u_{T}=\mu g / \bar{\eta}$ and introducing a new variable $\widetilde{v}=\widetilde{u}-$ $\mu g /(\bar{\eta} s)$ some algebraical manipulation leads to

$$
\begin{aligned}
\widetilde{v}(s)= & v_{0} \frac{s+\alpha}{(s+\alpha)^{2}+\omega^{2}}+\left[\left(1-\bar{\eta}^{*} / \bar{\eta}\right) \frac{\mu g}{\omega m}\right. \\
& \left.+\left(\frac{\bar{E}}{\bar{\eta}}-\frac{\bar{\eta}^{*}}{m}\right) \frac{v_{0}}{2 \omega}\right] \frac{\omega}{(s+\alpha)^{2}+\omega^{2}},
\end{aligned}
$$

with $\alpha \equiv\left(\bar{E} / \bar{\eta}+\bar{\eta}^{*} / m\right) / 2, \quad \omega=\sqrt{\bar{E} / m-\alpha^{2}}$, and $v_{0}=$ $u(0)-\mu g / \bar{\eta}$. The above equation can be instantly transformed back into the time domain leading to a similar expression as Eq. (A8). The particular case $v_{0}=0$ of this inverse transform reads

$$
\dot{x}(t)=u_{T}+e^{-\alpha t}\left[\left(1-\frac{\bar{\eta}^{*}}{\bar{\eta}}\right) \frac{\mu g}{\omega m}\right] \sin \omega t .
$$

Note that now the oscillational term has its smallest amplitude $A$. When we follow a similar line of reasoning as we did in Sec. IV B and compute the ratio of amplitude and terminal velocity we obtain

$$
\frac{A}{u_{T}}=\left(1-\frac{\bar{\eta}^{*}}{\bar{\eta}}\right) \frac{\mu g}{m \omega} \frac{\bar{\eta}}{\mu g}=\frac{\bar{\eta}-\bar{\eta}^{*}}{m \omega} \approx \frac{\bar{\eta}-\bar{\eta}^{*}}{m \omega_{0}} .
$$

This ratio can be small if $\bar{\eta}^{*} \approx \bar{\eta}$, which equation for both eM and mKV lead to $\eta^{*} \approx \eta^{*}+\eta$, which means that $\eta$ and $\eta^{*}$ both need to be small (compared to $m \omega_{0}$ ). What does this imply for the ratio of the damping time $\tau=1 / \alpha$ and the oscillation period $T=2 \pi / \omega$ ? We have

$$
2 \pi \frac{\tau}{T}=\frac{\omega}{\alpha}=\frac{2 \omega}{\left(\bar{E} / \bar{\eta}+\bar{\eta}^{*} / m\right)} \approx \frac{2 \omega \bar{\eta}}{\bar{E}}=\frac{2 \omega \bar{\eta}}{m \omega_{0}^{2}} \approx \frac{2 \bar{\eta}}{m \omega_{0}},
$$


where, for the first approximate equality, we used that $\bar{\eta}$ and $\bar{\eta}^{*}$ are both small and, for the second one, that $\omega$ is of the same order as $\omega_{0}=\sqrt{\bar{E} / m}$. Now both $A / u_{T}$ and $\tau / T$ are of order $\bar{\eta} /\left(m \omega_{0}\right)$. So if $A / u_{T}$ is small (as it should be in order to have the oscillations with amplitude smaller than drift velocity we observe), then so should $\tau / T$. And if $\tau / T$ is small, this means that the oscillation will damp out well within a single period, which contradicts the observations. Therefore, also the eM and $\mathrm{mKV}$ models are not capable of describing the observed bulk oscillations.
[1] N. J. Wagner and J. F. Brady, Phys. Today 62, 27 (2009).

[2] H. Barnes, J. Rheol 33, 329 (1989).

[3] A. Fall, N. Huang, F. Bertrand, G. Ovarlez, and D. Bonn, Phys. Rev. Lett. 100, 018301 (2008).

[4] E. Brown and H. M. Jaeger, Phys. Rev. Lett. 103, 086001 (2009).

[5] E. Brown, N. Forman, C. Orellana, H. Zhang, B. Maynor, D. Betts, J. M. De Simone, and H. Jaeger, Nature Materials 9, 220 (2010).

[6] C. Bonnoit, T. Darnige, E. Clement, and A. Lindner, J. Rheol. 54, 65 (2010).

[7] E. Brown and H. Jaeger, J. Rheol. 56, 875 (2012).

[8] A. J. Liu and S. R. Nagel, Nature 396, 21 (1998).

[9] M. van Hecke, J. Phys.: Cond. Matt. 22, 033101 (2010).

[10] S. Torquato, T. M. Truskett, and P. G. Debenedetti, Phys. Rev. Lett. 84, 2064 (2000)

[11] S. R. Williams and A. P. Philipse, Phys. Rev. E 67, 051301 (2003).

[12] J. Willett, Cereal Chem. 78, 64 (2001).

[13] H. He, Z. Guo, P. Stroeven, M. Stroeven, and L. J. Sluys, Mater. Charact. 60, 1082 (2009).

[14] C. Bonnoit, J. Lanuza, A. Lindner, and E. Clement, Phys. Rev. Lett. 105, 108302 (2010).
[15] A. Fall, F. Bertrand, G. Ovarlez, and D. Bonn, J. Rheol. 56, 575 (2012).

[16] E. B. White, M. Chellamuthu, and J. Rothstein, Rheol. Acta 49, 119 (2010).

[17] F. S. Merkt, R. D. Deegan, D. I. Goldman, E. C. Rericha, and H. L. Swinney, Phys. Rev. Lett. 92, 184501 (2004).

[18] R. D. Deegan, Phys. Rev. E 81, 036319 (2010).

[19] H. Ebata, S. Tatsumi, and M. Sano, Phys. Rev. E 79, 066308 (2009).

[20] H. Ebata and M. Sano, Phys. Rev. Lett. 107, 088301 (2011).

[21] S. von Kann, J. H. Snoeijer, D. Lohse, and D. van der Meer, Phys. Rev. E 84, 060401 (2011).

[22] B. Liu, M. Shelley, and J. Zhang, Phys. Rev. Lett. 105, 188301 (2010)

[23] N. Abaid, D. Adalsteinsson, A. Agyapong, and R. McLaughlin, Phys. Fluids 16, 1567 (2004).

[24] B. Akers and A. Belmonte, J. Non-Newtonian Fluid Mech. 135, 97 (2006).

[25] M. Arigo and G. McKinley, J. Rheol. 41, 103 (1997).

[26] D. Lohse, R. Rauhé, R. Bergmann, and D. van der Meer, Nature 432, 689 (2004).

[27] S. Waitukaitis and H. Jaeger, Nature 487, 205 (2012). 